\title{
ESTILOS EXPLICATIVOS Y AFECTO NEGATIVO
}

\author{
Pilar SAnjuÁn, AnA M. PÉrez-GarcíA, BeAtriz Rueda y M. Ángeles Ruiz \\ Facultad de Psicología, Universidad Nacional de Educación a Distancia, Madrid
}

\begin{abstract}
Resumen: El estilo explicativo negativo se refiere a la tendencia relativamente estable a explicar las situaciones negativas mediante causas internas, estables y globales. Gran cantidad de estudios han relacionado este estilo con depresión clínica o síntomas depresivos. Algunos estudios también le han asociado con ansiedad y otras emociones negativas. El principal objetivo del presente trabajo fue estudiar las relaciones entre el estilo explicativo negativo, afecto negativo y síntomas de ansiedad y depresión. Los resultados mostraron, tal como se esperaba, que cuando se controlaba el efecto del afecto negativo, las relaciones entre este estilo explicativo y los síntomas de ansiedad y depresión desaparecían, mientras que cuando se controlaban los síntomas de ansiedad y depresión la asociación entre el estilo explicativo y afecto negativo se mantenía. Se concluye que el estilo explicativo negativo no es específico de la depresión, sino que constituye un estilo cognitivo asociado al malestar psicológico.
\end{abstract}

Palabras clave: Estilo explicativo negativo, ansiedad, depresión, afecto negativo.

\section{Explanatory styles and negative affect}

\begin{abstract}
The negative explanatory style refers to the relatively stable tendency to explain negative events by internal, stable, and global causes. This style has been widely related to clinical depression or depressive symptoms. Some studies have also shown an association with anxiety and other negative emotions. The first goal of the present work was to study the relationships between negative explanatory style, negative affect and symptoms of anxiety and depression. As expected, the results showed that when negative affect was controlled, the association between this explanatory style and symptoms of anxiety and depression disappeared, whereas when symptoms of anxiety and depression were controlled the relation between the explanatory style and negative affect was maintained. It is concluded that negative explanatory style is not specific to depression, but it constitutes a cognitive style associated with psychological distress.
\end{abstract}

Keywords: Negative explanatory style, anxiety, depression, negative affect.

\section{INTRODUCCIÓN}

El estilo explicativo o atribucional negativo se define como una tendencia relativamente estable a explicar las situaciones negativas o fracasos mediante causas internas (la causa es algo personal del individuo), estables (la causa se mantiene a lo largo del tiempo) y globales (la causa afecta a diferentes áreas de la vida).

Este estilo comenzó a estudiarse cuando el modelo reformulado de indefensión aprendi-

Recibido 19 julio 2007; aceptado 20 noviembre 2007.

Correspondencia: Pilar Sanjuán, Dpto. Personalidad, Evaluación y Tratamiento Psicológicos, Facultad de Psicología, UNED, Juan del Rosal, 10, Ciudad Universitaria, 28040 Madrid. Correo-e: psanjuan@psi.uned.es da (Abramson, Seligman y Teasdale, 1978) le propuso como un factor de vulnerabilidad específico para desarrollar depresión cuando las personas se enfrentasen a situaciones estresantes o negativas en sus vidas. Desde entonces, numerosos estudios, llevados a cabo, tanto desde este modelo reformulado, como desde la teoría de la desesperanza (Abramson, Metalsky y Alloy, 1989; Abramson, Alloy y Metalsky, 1995; Abramson, Alloy, Metalsky, Joiner y Sandín, 1997), que supone un desarrollo posterior del mismo, han mostrado que

Trabajo realizado por el grupo de investigación «Personalidad y Salud» (Ref.n ${ }^{\circ}$ : G59E47) gracias a la financiación de la Subdirección General de Proyectos de Investigación del Ministerio de Educación y Ciencia (Referencia Proyecto: SEJ2004-03834) 
las personas con un estilo explicativo negativo, con respecto a las que se caracterizan por un estilo explicativo positivo (polo opuesto de la dimensión o tendencia a explicar las situaciones negativas a través de causas externas, inestables y específicas) presentan más síntomas depresivos cuando se enfrentan a situaciones problemáticas (Abela, 2001; Alloy y Clements, 1998; Alloy, Just y Panzarella, 1997; Dixon y Ahrens, 1992; Hankin y Abramson, 2002; Hankin, Abramson y Siler, 2001; Hilsman y Garber, 1995; Kneebone y Dunmore, 2004; Know y Laurenceau, 2002; Metalsky, Halberstadt y Abramson, 1987; Metalsky y Joiner, 1992, 1997; Sanjuán, 2007; Sanjuán y Magallares, 2006a, 2007, en prensa; Stiensmeier-Pelster, 1989; Sweeney, Anderson y Bailey, 1986; Vázquez, Jiménez, Saura y Avia, 2001).

Aunque la gran mayoría de los estudios han podido corroborar la hipótesis de partida, prácticamente todos han medido sólo síntomas depresivos, sin embargo, algunos trabajos, que han estudiado síntomas de ansiedad, también han encontrado que mantienen una relación con este estilo explicativo (Ahrens y Haaga, 1993; Camuñas, Cano-Vindel, Pérez-Nieto y González, 2002; Helton, Dember, Warm y Matthews, 2000; Jackson, Sellers y Peterson, 2002; Johnson y Miller, 1990; Kopecky, Sawyer y Behnke, 2004; Martin-Krumm, Sarrazin, Peterson y Famose, 2003; Mineka, Pury y Luten, 1995; Ralph y Mineka, 1998; Wang y Zhang, 2005).

Dado que ansiedad y depresión comparten algunos síntomas como el alto afecto negativo (Clark y Watson, 1991; Watson y Tellegen, 1985), y puesto que unos pocos estudios también han encontrado que el estilo explicativo negativo se asocia con otras emociones negativas como la ira y la hostilidad (Boman, Smith y Curtis, 2003; Camuñas et al., 1999; Sanjuán, Pérez García, Rueda y Ruiz, 2006), se podría especular sobre la idea de que el estilo atribucional negativo no fuera un factor asociado específicamente a la sintomatología depresiva sino que más bien pudiera constituir un factor de vulnerabilidad general para desarrollar malestar psicológico o emociones negativas.
En apoyo de esta sugerencia, y teniendo en cuenta, no sólo la presencia de sintomatología, sino también la de algún trastorno psicopatológico, el estilo explicativo negativo se ha asociado, por supuesto, con depresión clínica (Abramson et al., 2002; Alloy et al., 1999; Alloy et al., 2000; Alloy et al., 2006; Mongrain y Blackburn, 2005; Sturman, Mongrain y Kohn, 2006), pero también con trastornos de ansiedad (Alloy et al., 2006; Fresco, Alloy y Reilly-Harrington, 2006; Haeffel et al., 2003; Heimberg et al., 1989), trastornos de personalidad (Alloy et al., 1999; Ilardy y Craighead, 1999; Mongrain y Blackburn, 2005) y esquizofrenia (Fraguas et al., en prensa; Krstev, Jackson y Maude, 1999; Zimmerman, Coryell, Corenthal y Wilson, 1986).

El problema que se ha planteado sobre la especificidad es difícil de resolver ya que, salvo algunas excepciones (Ahrens y Haaga, 1993; Fresco et al., 2006; Johnson y Miller, 1990; Ralph y Mineka, 1998), los estudios sólo evalúan los síntomas de ansiedad o depresión, pero no miden ambos a la vez. El objetivo principal del presente trabajo fue estudiar, en una muestra no clínica compuesta de mujeres, las relaciones que mantiene el estilo atribucional negativo tanto con los síntomas de ansiedad y depresión, como con afecto negativo. De acuerdo, tanto con las evidencias presentadas previamente, como con las ideas sugeridas, esperábamos que el estilo explicativo negativo guardaría una relación directa con síntomas de ansiedad y depresión, así como con afecto negativo, sin embargo, considerábamos que, una vez controlado el efecto del resto de las variables, sólo se mantendría la relación entre el citado estilo y el afecto negativo.

\section{MÉTODO}

\section{Participantes}

Se empleó una muestra formada por 327 mujeres estudiantes universitarias de toda la geografía española, con un rango de edad entre 17 y 60 años y con una media de 35,4 años $(D T=9,96)$. 


\section{Medidas y procedimiento}

Los participantes contestaron a las siguientes medidas:

Cuestionario de Estilo Atribucional [Attributional Style Questionnaire, ASQ] (Peterson et al., 1982). Se evaluó con la adaptación española (Sanjuán y Magallares, 2006b; Sanjuán, Magallares, Pérez García, Rueda y Ruiz, 2007). Este cuestionario consta de 12 situaciones hipotéticas, 6 positivas (por ej., «obtiene Ud. un ascenso») y 6 negativas (por ej., «no puede realizar todo el trabajo que se espera de Ud.»). Los participantes contestan en cada una de estas 12 situaciones, el grado en que creen que la causa de la situación es interna, estable y global, mediante escalas tipo Likert de 7 puntos (de 1 a 7). Se puede obtener una puntuación por cada una de las dimensiones atribucionales (internalidad, estabilidad y globalidad) tanto para los sucesos positivos como para los negativos separadamente y dos puntuaciones globales, correspondientes a los estilos atribucionales para las situaciones positivas (EASP) y las negativas (EASN). Dado que los propósitos del estudio sólo se refieren a las relaciones entre el estilo explicativo presentado ante las situaciones negativas y diferentes medidas, sólo se empleó la puntuación en EASN, la cual alcanzó una fiabilidad adecuada en la presente muestra $(\alpha=0,77)$. Las puntuaciones elevadas en EASN indican tendencia a realizar atribuciones internas, estables y globales para las situaciones negativas, o estilo explicativo negativo, mientras que las puntuaciones bajas señalan la pre- disposición a explicar las situaciones negativas mediante causas externas, inestables y específicas (al que se ha denominado, por oposición, estilo explicativo positivo).

Escalas de Afecto Positivo y Negativo [Positive and Negative Affect Schedule, PANAS] (Watson, Clark y Tellegen, 1988). Se aplicó la versión española validada por Sandín et al. (1999). Esta escala consta de 20 items, 10 relativos a la dimensión de afecto positivo y otros 10 que miden afecto negativo. Los participantes contestan mediante escalas tipo Likert de 5 puntos el grado en que experimentan distintas emociones de manera habitual. De acuerdo con los objetivos del estudio, sólo se empleo la subescala de afecto negativo, que en la muestra actual obtuvo una fiabilidad muy elevada $(\alpha=0,88)$

Escala de Ansiedad y Depresión Hospitalaria [Hospital Anxiety and Depression Scale, HADS] (Zigmond y Snaith, 1983). Se midieron mediante la versión española (Rueda, 2004). Esta escala consta de 14 items, la mitad de los cuales hacen referencia a síntomas depresivos y la otra mitad a síntomas de ansiedad. Mediante escalas tipo Likert de 5 puntos se indica la frecuencia con que se han experimentado dichos síntomas durante las dos semanas anteriores. Los coeficientes de consistencia interna obtenidos en el presente estudio fueron de $0,84 \mathrm{y}$ de 0,83 para ansiedad y depresión respectivamente.

\section{RESULTADOS}

En la Tabla 1 aparecen los estadísticos descriptivos de todas las variables analizadas en el estudio para la muestra total.

Tabla 1. Estadísticos descriptivos de las variables del estudio en la muestra total y los grupos formados

\begin{tabular}{lccc}
\hline & $\begin{array}{c}\text { Total } \\
(n=327)\end{array}$ & $\begin{array}{c}\text { Estilo Explicativo Positivo } \\
(n=86)\end{array}$ & $\begin{array}{c}\text { Estilo Explicativo Negativo } \\
(n=82)\end{array}$ \\
\hline Media $(D T)$ & Media $(D T)$ & Media $(D T)$ \\
\hline Síntomas de Ansiedad & $12,85(3,78)$ & $11,32(2,87)$ & $14,03(4,22)$ \\
Síntomas de Depresión & $10,82(3,75)$ & $9,55(3,33)$ & $11,96(4,19)$ \\
Afecto Negativo & $17,53(5,87)$ & $14,65(3,46)$ & $20,15(7,32)$ \\
EASN & $69,99(13,17)$ & $53,34(7,13)$ & $85,96(7,53)$ \\
\hline
\end{tabular}

Nota $:$ EASN = Estilo Atribucional para las Situaciones Negativas. Cuanto más alta es la puntuación se indican atribuciones más internas, estables y globales. 
Como primer paso, calculamos las correlaciones entre el estilo atribucional para las situaciones negativas (EASN) y las demás variables, que como puede verse en la Tabla 2 fueron todas altamente significativas. Además, también se obtuvieron las correlaciones parciales controlando alguna o varias de las variables criterio consideradas. De esta manera, se comprobó que controlando los síntomas depresivos, la correlación mantenida con los síntomas de ansiedad, aunque disminuía bastante de intensidad, seguía siendo significativa. Sin embargo, cuando se controló el efecto de los síntomas de ansiedad, la correlación con síntomas de depresión disminuía hasta perder la significación. De la misma manera, cuando se controlaba el efecto del afecto negativo, las correlaciones obtenidas tanto con los síntomas de ansiedad como de depresión fueron irrelevantes. Sin embargo, controlando los síntomas de ansiedad y depresión, tanto por separado como conjuntamente, la correlación establecida con afecto negativo seguía manteniendo su significación.

Como siguiente paso se formaron grupos extremos en el EASN, utilizando como criterio la selección de las personas con puntuaciones altas (por encima del tercer cuartil) o participantes con estilo explicativo negativo $(n=$ 86), y las que tenían puntuaciones bajas (por debajo del primer cuartil) o personas con estilo explicativo positivo $(n=82)$. Los grupos estaban bien formados ya que mostraban diferencias altamente significativas en la variable de clasificación $[F(1,167)=828,86, p<0,000]$.
En la Tabla 1 pueden verse las medias y las desviaciones típicas de las variables analizadas en función de los grupos.

Se realizaron sucesivos análisis de varianza, todos ellos de un factor manipulado entre-sujetos (altos en EASN o estilo explicativo negativo $v s$. bajos en EASN o estilo explicativo positivo), empleando como variables dependientes tanto los síntomas de ansiedad y depresión, como el afecto negativo. El primero se llevó a cabo sin emplear ningún covariante y en los posteriores se utilizaron como covariantes cada una de las variables analizadas. En la Tabla 3 se incluye un resumen de los efectos encontrados y como puede observarse, cuando no se empleó covariante, encontramos que las personas con un estilo explicativo negativo presentaban más síntomas de ansiedad y depresión, así como más afecto negativo que aquellas con un estilo explicativo positivo. Los efectos sobre los síntomas de depresión desaparecían cuando se controlaba ansiedad, pero permanecían los efectos sobre el afecto negativo. Cuando se empleaba de covariante los síntomas de depresión, los efectos sobre los síntomas de ansiedad y el afecto negativo se mantenían. Después de controlar los síntomas de ansiedad y depresión, seguían existiendo efectos sobre el afecto negativo, pero cuando era al revés, es decir, cuando se empleaba el afecto negativo como covariante, los efectos sobre los síntomas de ansiedad y depresión desaparecían.

Tabla 2. Correlaciones entre el estilo atribucional para las situaciones negativas (EASN) y las demás variables del estudio

\begin{tabular}{|c|c|c|c|c|c|}
\hline & $\begin{array}{c}\text { Correlación } \\
\text { bivariada }\end{array}$ & $\begin{array}{c}\text { Correlación } \\
\text { parcial (controlado } \\
\text { sint. de ansiedad) }\end{array}$ & $\begin{array}{c}\text { Correlación } \\
\text { parcial (controlado } \\
\text { sint. de depresión) }\end{array}$ & $\begin{array}{c}\text { Correlación } \\
\text { parcial (controlado } \\
\text { sint. de ansiedad } \\
\text { y depresión) }\end{array}$ & $\begin{array}{c}\text { Correlación } \\
\text { parcial (controlado } \\
\text { afecto negativo) }\end{array}$ \\
\hline Síntomas de Ansiedad & $0,29 * *$ & - & $0,17 *$ & - & 0,08 \\
\hline Síntomas de Depresión & $0,26 * *$ & 0,07 & - & - & 0,09 \\
\hline Afecto Negativo & $0,39 * *$ & $0,28 * *$ & $0,32 * *$ & $0,28 * *$ & - \\
\hline
\end{tabular}

$* p<0.003 * * p<0.000$ 
Tabla 3: Resumen de resultados obtenidos en los diferentes diseños de ANOVA

\begin{tabular}{lccc}
\hline COVARIANTE & VARIABLE & $F(1,167)$ & $p$ \\
\hline \multirow{2}{*}{ Ninguno } & Síntomas de depresión & 17,03 & 0,000 \\
& Síntomas de ansiedad & 23,52 & 0,000 \\
& Afecto negativo & 38,17 & 0,000 \\
\hline \multirow{2}{*}{ Síntomas de ansiedad } & Síntomas de depresión & 1,01 & $>0,3$ \\
& Afecto negativo & 14,89 & 0,000 \\
\hline \multirow{2}{*}{ Síntomas de depresión } & Síntomas de ansiedad & 6,89 & 0,009 \\
& Afecto negativa & 22,27 & 0,000 \\
\hline Síntomas de ansiedad y depresión & Afecto negativo & 14,89 & 0,000 \\
\hline Afecto negativo & Síntomas de depresión & 2,88 & $>0,1$ \\
& Síntomas de ansiedad & 1,98 & $>0,2$ \\
\hline
\end{tabular}

\section{DISCUSIÓN}

La primera conclusión que se puede deducir de los resultados obtenidos es que el estilo explicativo negativo no mantiene una asociación específica con los síntomas de depresión, puesto que también se relaciona con síntomas de ansiedad y afecto negativo. Sería más adecuado hablar, por tanto, de un factor que confiere vulnerabilidad para experimentar malestar psicológico.

Además, gracias a los análisis realizados se ha podido descubrir que los síntomas depresivos, que son en los que más se ha centrado la investigación hasta la fecha, pierden la asociación mantenida cuando se controla el efecto de los síntomas de ansiedad. Sin embargo, la relación con los síntomas de ansiedad, que sólo ha ocupado un lugar colateral en la investigación, sí que se mantiene, aunque se controlen los efectos de los síntomas depresivos. Estos hechos, interpretados conjuntamente, podrían estar indicando que la relación entre el estilo explicativo negativo y los síntomas de ansiedad es más robusta. Esta sugerencia estaría en la línea de argumentación defendida por algunos autores que mantienen que el estilo explicativo negativo es más característico de la ansiedad que de la depresión (Ahrens y Haaga, 1993; Fresco et al., 2006; Mineka et al., 1995).

De todas formas es necesario matizar más esta conclusión anterior, puesto que cuando se controla el afecto negativo se pierde la asocia- ción entre este estilo y ansiedad y depresión, pero, sin embargo, cuando se controla ansiedad y depresión conjuntamente, no se pierde la relación que se mantenía con afecto negativo. A la luz de estos datos, parece que el estilo explicativo negativo se relacionaría con la experiencia frecuente de diferentes emociones negativas, entre las que se pueden incluir los sentimientos depresivos y ansiógenos, pero con una ponderación moderada en comparación con otras emociones negativas, que tendrían, en conjunto, un mayor peso.

Estos resultados ponen de manifiesto que al limitar el estudio de las relaciones que mantiene el estilo explicativo negativo al análisis de los síntomas de depresión o de ansiedad se restringen las posibilidades de descubrir el verdadero rango de acción de este importante factor de riesgo. En el ámbito de lo psicológico, sería muy recomendable por tanto, incluir medidas de otras emociones negativas o de afecto negativo en general cuando se estudian los efectos del estilo explicativo negativo. En la esfera de lo psicopatológico, también sería necesario el estudio de diversos cuadros clínicos, pues ya existe alguna evidencia, tal como se ha señalado previamente, sobre la asociación de este estilo explicativo, no sólo con trastornos depresivos (Abramson et al., 2002; Alloy et al., 1999; Alloy et al., 2000; Alloy et al., 2006; Mongrain y Blackburn, 2005; Sturman et al., 2006) o de ansiedad (Alloy et al., 2006; Fresco et al., 2006; Haeffel et al., 2003; Heimberg et al., 1989), 
sino también con trastornos de personalidad (Alloy et al., 1999; Ilardy y Craighead, 1999; Mongrain y Blackburn, 2005) y esquizofrenia (Fraguas et al., en prensa; Krstev et al., 1999; Zimmerman et al., 1986).

Además, y en defensa del argumento sobre el amplio espectro de acción de este estilo explicativo, en el área de la salud física, también se ha encontrado que, en comparación con las personas caracterizadas por un estilo explicativo positivo, las personas con estilo explicativo negativo informan de más enfermedades (Jackson et al., 2002; Peterson, 1988), realizan más visitas al médico (Peterson, 1988; Peterson y De Ávila, 1995), padecen más enfermedades (Peterson, Seligman y Vaillant, 1988), están más tiempo enfermos (Dykema, Bergbower y Peterson, 1995), su tiempo de supervivencia después del diagnóstico de cáncer o enfermedad cardiaca es menor (Buchanan, 1995; Peterson, 1995) y tienen un porcentaje de mortalidad más elevado (Peterson, Seligman, Yurko, Martín y Friedman, 1998).

Para finalizar habría que añadir que, puesto que la muestra empleada sólo es de mujeres, y dado que éstas informan de más síntomas de ansiedad y depresión que los varones, sería aconsejable que se replicaran estos resultados también con muestras de varones. También sería recomendable que se intentara corroborar estos resultados mediante estudios longitudinales que permitieran estudiar el posible papel causal del estilo explicativo negativo en el inicio, desarrollo y mantenimiento del malestar psicológico.

\section{REFERENCIAS}

Abela, J. (2001). The hopelessness theory of depression: A test of the diathesis-stress and causal mediation components in third and seventh grade children. Journal of Abnormal Child Psychology, 29, 241-254.

Abramson, L.Y., Alloy, L., Hogan, M., Whitehouse, W., Donovan, P., Rose, D., Panzarella, C., y Raniere, D. (2002) Cognitive vulnerability to depression: Theory and evidence. En R.L. Leahy y E.T. Dowd (Eds.), Clinical advances in cognitive psychotherapy: Theory and applications (pp. 75-92). Nueva York: Springer Publishing Co.

Abramson, L.Y., Alloy, L.B., y Metalsky, G.I. (1995). Hopelessness depression. En G.M. Buchanan y M.E.P.
Seligman (Eds.), Explanatory Style (pp.113-134). Hillsdale, N.J.: Erlbaum.

Abramson, L.Y., Alloy, L.B., Metalsky, G.I., Joiner, T.E., y Sandín, B. (1997). Teoría de la depresión por desesperanza: Aportaciones recientes. Revista de Psicopatología y Psicología Clínica, 2, 211-222.

Abramson, L.Y., Metalsky, G.I., y Alloy, L.B. (1989). Hopelessness depression: A theory-based subtype of depresión. Psychological Review, 96, 358-372.

Abramson, L.Y., Seligman, M.E.P., y Teasdale, D.C. (1978). Learned helplessness in humans: Critique and reformulation. Journal of Abnormal Psychology, 87, 49-74.

Ahrens, A. H., y Haaga, D.A.F. (1993). The specificity of attributional style and expectations to positive and negative affectivity, depression and anxiety. Cognitive Therapy and Research, 17, 83-98.

Alloy, L., Abramson, L.Y., Hogan, M., Whitehouse, W., Rose, D., Robinson, M., y Kim, R. (2000). The Temple-Wisconsin Cognitive Vulnerability to Depression Project: Lifetime, history of axis I psychopathology individuals at high and low cognitive risk for depression. Journal of Abnormal Psychology, 109, 403-417.

Alloy, L., Abramson, L.Y., Whitehouse, W., Hogan, M., Panzarella, C., y Rose, D. (2006). Prospective incidence of first onsets and recurrences of depression in individuals at high and low cognitive risk for depression. Journal of Abnormal Psychology, 115, 145-156.

Alloy, L., Abramson, L.Y., Whitehouse, W., Hogan, M., Tashman, N.A., Steinberg, D., Rose, D., y Donovan, P. (1999). Depressogenic cognitive styles: Predictive validity, information processing and personality characteristics, and developmental origins. Behaviour Research and Therapy, 37, 503-531.

Alloy, L.B., y Clements, C.M. (1998). Hopelessness theory of depression: Tests of the symptom component. Cognitive Therapy and Research, 22, 303-335.

Alloy, L.B., Just, N., y Panzarella, C. (1997). Attributional style, daily life events, and hopelessness depression: Subtype validation by prospective variability and specificity of symptoms. Cognitive Therapy and Research, 21, 321-344.

Boman, P., Smith, D.C., y Curtis, D. (2003). Effects of pessimism and explanatory style on development of anger in children. School Psychology International, 24, 80-94.

Buchanan, G.M. (1995). Explanatory style and coronary hearth disease. En G.M. Buchanan y M.E.P. Seligman (Eds.), Explanatory style (pp. 225-232). Hillsdale, N.J.: Erlbaum.

Camuñas, N., Cano-Vindel, A., Pérez Nieto, M., y González Ordi, H. (2002). Inventario de atribuciones causales ante exámenes -IACE-: Propiedades psicométricas. Ansiedad y Estrés, 8, 183-192. 
Camuñas, N., Pérez Nieto, M., Ferrándiz, P., MiguelTobal, J., Cano-Vindel, A., e Iruarrizaga, I. (1999). Ansiedad, depresión e ira: relaciones entre el estilo atribucional y las emociones negativas. Ansiedad y Estrés, 5, 175-189.

Clark, L., y Watson, D. (1991). Tripartite model of anxiety and depression. Psychometric and taxonomic implications. Journal of Abnormal Psychology, 100, 316-336.

Dixon, J.F., y Ahrens, A.H. (1992). Stress and attributional style as predictor of self-reported depression in children. Cognitive Therapy and Research, 16, 623-634.

Dykema, J., Bergbower, K., y Peterson, C. (1995). Pessimistic explanatory style, stress, and illness. Journal of Social and Clinical Psychology, 14, 357-371.

Fraguas, D., Mena, A., Franco, C., Martín-Blas, M., Nugent, K y Rodríguez-Solano, J. (en prensa). Attributional style, symptomatology and awareness of illness in schizophrenia. Psychiatry Research.

Fresco, D.M., Alloy, L.B., y Reilly-Harrington, N. (2006). Association of attributional style for negative and positive events and the occurrence of life events with depression and anxiety. Journal of Social and Clinical Psychology, 25, 1140-1159

Haeffel, G. Abramson, L., Voelz, Z., Metalsky, G., Halberstadt, L., y Dykema, B. (2003). Cognitive vulnerability to depression and lifetime history or axis I psychopathology: A comparison of negative cognitive styles and dysfunctional attitudes. Journal of Cognitive Psychotherapy, 17, 3-22.

Hankin, B.L., y Abramson, L.Y. (2002). Measuring cognitive vulnerability in adolescence: Reliability, validity and gender differences. Journal of Clinical Child and Adolescent Psychology, 31, 491-504.

Hankin, B.L., Abramson, L.Y., y Siler, M. (2001). A prospective test of the hopelessness theory of depression in adolescence. Cognitive Therapy and Research, 25, 607-632.

Heimberg, R., Klosko, J., Dodge, C., Shadick, R., Becker, R., y Barlow, D. (1989). Anxiety disorders, depression, and attributional style: A further test of the specificity of depressive attributions. Cognitive Therapy and Research, 13, 21-36.

Helton, W.S., Dember, W.N., Warm, J.S., y Matthews, G. (2000). Optimism, pessimism, and false failure feedback effects on vigilance performance. Current Psychology, 18, 311-325.

Hilsman, R., y Garber, J. (1995). A test the cognitive diathesis-stress model of depression in children: academic stressors, attributional style, perceived competence, and control. Journal of Personality and Social Psychology, 2, 370-380.

Ilardi, S., y Craighead, W. (1999). The relationship between personality pathology and dysfunctional cog- nitions in previously depressed adults. Journal of Abnormal Psychology, 108, 51-57.

Jackson, B., Sellers, R.M., y Peterson, C. (2002). Pessimistic explanatory style moderates the effects of stress on physical illness. Personality and Individual Differences, 32, 567-573.

Johnson, J., y Miller, S. (1990). Attributional, life event, and affective predictors of onset of depression, anxiety, and negative attributional style. Cognitive Therapy and Research, 14, 417-430.

Kneebone, I.I., y Dunmore, E. (2004). Attributional style and symptoms of depression in persons with multiple sclerosis. International Journal of Behavioral Medicine, 11, 110-115.

Know, P., y Laurenceau, J.P. (2002). A longitudinal study of the hopelessness theory of depression: Testing the diathesis-stress model within a differential reactivity exposure framework. Journal of Clinical Psychology, $58,1305-1321$

Kopecky, C., Sawyer, C., y Behnke, R. (2004). Sensitivity to punishment and explanatory style as predictors of public speaking state anxiety. Communication Education, 53, 281-285.

Krstev, H., Jackson, H y Maude, D. (1999). An investigation of attributional style in first-episode psychosis. British Journal of Clinical Psychology, 38, 181-194.

Martin-Krumm, C., Sarrazin, P., Peterson, C., y Famose, J. (2003). Explanatory style and resilience after sport failure. Personality and Individual Differences, 35 , 1685-1697.

Metalsky, G.I., Halberstadt, L.J., y Abramson, L.Y. (1987). Vulnerability to depressive mood reactions: Toward a more powerful test of the diathesis-stress and causal mediation components of the reformulated theory of depression. Journal of Personality and Social Psychology, 52, 386-393.

Metalsky, G.I., y Joiner, T.E. (1992). Vulnerability to depressive symptomatology: A prospective test of the diathesis-stress and causal mediation components of the hopelessness theory of depression. Journal of Personality and Social Psychology, 63, 667-675.

Metalsky, G.I., y Joiner, T.E. (1997). The Hopelessness Depression Symptom Questionnaire. Cognitive Therapy and Research, 21, 359-384.

Mineka, S., Pury, C.L., y Luten, A.G. (1995). Explanatory style in anxiety and depression. En G.M. Buchanan y M.E.P. Seligman (Eds.), Explanatory style (pp. 135157). Hillsdale, N.J.: Erlbaum.

Mongrain, M., y Blackburn, S. (2005). Cognitive vulnerability, lifetime risk, and the recurrence of mayor depression in graduate students. Cognitive Therapy and Research, 29, 747-768.

Peterson, C. (1988). Explanatory style as a risk factor for illness. Cognitive Therapy and Research, 12, 119-132. 
Peterson, C. (1995). Explanatory style and health. En G.M. Buchanan y M.E.P. Seligman (Eds.), Explanatory style (pp. 233-2246). Hillsdale, N.J.: Erlbaum.

Peterson, C., y de Ávila, M. (1995). Optimistic explanatory style and the perception of health problems. Journal of Clinical Psychology, 51, 128-132.

Peterson, C., Seligman, M.E.P., y Vaillant, G.E. (1988). Pessimistic explanatory style is a risk factor for physical illness: A thirty-five-year longitudinal study. Journal of Personality and Social Psychology, 55, 23-27.

Peterson, C., Seligman, M.E.P., Yurko, K.H., Martin, L.R., y Friedman, H.S. (1998). Catastrophizing and untimely death. Psychological Science, 9, 127-130.

Peterson, C., Semmel, A., Baeyer, C., Abramson, L.Y., Metalsky, G.I., y Seligman, M.E.P. (1982). The Attributional Style Questionnaire. Cognitive Therapy and Research, 6, 287-300.

Ralph, J.A., y Mineka, S. (1998). Attributional style and self-esteem: The prediction of emotional distress following a midterm exam. Journal of Abnormal Psychology, 107, 203-215.

Rueda, B. (2004). Competencia percibida y salud: aproximación general y aplicación a la enfermedad cardiovascular. Tesis doctoral no publicada. Madrid: UNED.

Sandin, B., Chorot, P., Lostao, L., Joiner, T.E., Santed, M.A., y Valiente, R.M. (1999). Escalas PANAS de afecto positivo y negativo: Validación factorial y convergencia transcultural. Psicothema, 11, 37-51.

Sanjuán, P. (2007). Estilos explicativos, bienestar psicológico y salud. Ansiedad y Estrés, 13, 203-214.

Sanjuán, P., y Magallares, A. (2006a). Estilo atributivo negativo, sucesos vitales y sintomatología depresiva. Revista de Psicopatología y Psicología Clínica, 11, 91-98.

Sanjuán, P., y Magallares, A. (2006b). La relación entre optimismo disposicional y estilo atribucional y su capacidad predictiva en un diseño longitudinal. Revista de Psicología General y Aplicada, 59, 71-89.

Sanjuán, P., y Magallares, A. (2007). Estilos explicativos y estrategias de afrontamiento. Clínica y Salud, 18, 83-98.

Sanjuán, P., y Magallares, A. (En prensa). Coping strategies as link between optimistic explanatory styles and well-being. En T. Freire (Ed.), Understanding positive life. Research and practice on Positive Psychology. Lisboa: Climepsi Editores.
Sanjuán, P. Magallares, A., Pérez García, A.M., Rueda, B., y Ruiz, M.A. (2007). Study of construct validity of Attributional Style Questionnaire in a Spanish sample. Trabajo presentado en la IX European Conference on Psychological Assessment. Tesalónica (Grecia), 3-6 de Mayo.

Sanjuán, P., Pérez García, A., Rueda, B., y Ruiz, M.A. (2006). Estilos explicativos y salud. Trabajo presentado en la VI Semana de Investigación de la Facultad de Psicología de la UNED. Madrid, 20-24 de Noviembre.

Stiensmeier-Pelster, J. (1989). Attributional style and depressive mood reactions. Journal of Personality, 57, 581-599.

Sturman, E., Mongrain, M., y Kohn, P. (2006). Attributional style as a predictor of hopelessness depression. Journal of Cognitive Psychotherapy, 20, 447-458.

Sweeney, P.D., Anderson, K., y Bailey, S. (1986). Attributional style in depression: A meta-analytic review. Journal of Personality and Social Psychology, 50, 974-991.

Vázquez, C., Jiménez, F., Saura, F., y Avia, M.D. (2001). The importance of «importance»: A longitudinal-stress model of depression measuring the importance of the attributions and the impact of stressor. Personality and Individual Differences, 31, 205-214.

Wang, C., y Zhang, N. (2005). Personality correlates to attributional style in undergraduates. Chinese Journal of Clinical Psychology, 13, 53-54.

Watson, D., Clark, L.A., y Tellegen, A. (1988). Development and validation of brief measures of positive and negative affect. The PANAS scales. Journal of Personality and Social Psychology, 54, 1063-1070.

Watson, D., y Tellegen, A. (1985). Toward a consensual structure of mood. Psychological Bulletin, 98, 219235.

Zigmond, A.S., y Snaith, R.P. (1983). The Hospital Anxiety and Depression Scale. Acta Psychiatrica Scandinavica, 67, 361-370.

Zimmerman, M., Coryell, W., Corenthal, C., y Wilson, S. (1986). Dysfunctional attitudes and attribution style in healthy controls and patients with schizophrenia, psychotic depression, and non-psychotic depression. Journal of Abnormal Psychology, 95, 403-405. 